\title{
Distended oesophagus as a cause of bilateral recurrent laryngeal nerve palsy
}

\author{
L.S.P. Wickramasinghe, ${ }^{1}$ C.R. Chowdhury, ${ }^{2}$ S.S. Pillai ${ }^{2}$ and S. Ghosh ${ }^{1}$ \\ ${ }^{1}$ Department of Medicine for the Elderly, District General Hospital, Kayll Road, Sunderland SR4 7TP and \\ ${ }^{2}$ Royal Infirmary, Sunderland SR2 7JE, UK.
}

\begin{abstract}
Summary: We report an unusual case of bilateral recurrent laryngeal nerve palsy caused by gross oesophageal distension due to achalasia.
\end{abstract}

\section{Introduction}

Stridor resulting from bilateral recurrent laryngeal nerve palsy secondary to gross oesophageal distension is most unusual. We describe such an event in an elderly female patient.

\section{Case report}

An 85 year old female patient was admitted in acute respiratory distress. She had 6 months history of intermittent dysphagia for solids and liquids with regurgitation and weight loss of about $7 \mathrm{~kg}$. Four days before admission she had developed hoarseness accompanied by progressive dyspnoea and stridor. She had considerable inspiratory stridor with respiratory distress both in the sitting and in the lying position. There was no thyroid enlargement, lymphadenopathy or any other swelling in the neck. She was cachectic and cyanosed. A plain chest X-ray (Figure 1) and thoracic inlet view showed widening of superior mediastinum (with compressed tracheal air column) and clear lung fields. Indirect laryngoscopy showed bilateral adducted, immobile but passively mobile and morphologically normal vocal cords. Emergency tracheostomy was performed to relieve the airway obstruction and a Size 6 Shiley tracheostomy tube was introduced. During tracheostomy tracheal lumen was found to be narrowed by extrinsic pressure along the posterior wall but the mucosa was normal.

Subsequent oesophagogastroscopy showed a profoundly distended oesophagus filled with food particles. On complete evacuation of the oesophagus a constricted but easily negotiable lower

Correspondence: L.S.P. Wickramasinghe, M.R.C.P.(UK), D.G.M.(UK).

Accepted: 7 June 1988 oesophageal sphincter was found. Oesophageal and gastric mucosa appeared normal. Histology of mucosal biopsies showed no evidence of malignancy. Barium swallow examination showed profoundly distended oesophagus and slow flow into the stomach across a smoothly tapered gastrooesophageal junction, confirming achalasia.

The patient had no recurrence of stridor or 0 respiratory distress and on extubation both vocal

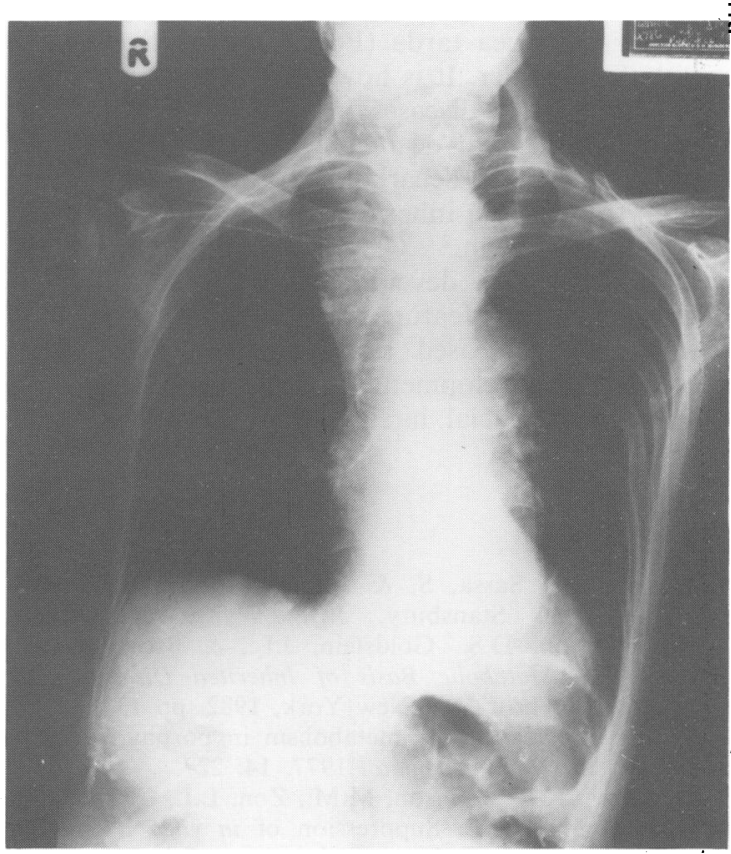

Figure 1 Plain chest X-ray showing superior mediastinal widening due to distended oesophagus filled with food particles and air.

(C) The Fellowship of Postgraduate Medicine, 1988 
cords were seen to abduct. Indirect laryngoscopy 4 days later showed normal position and movement of vocal cords.

\section{Discussion}

Upper airways obstruction due to non-traumatic bilateral recurrent laryngeal nerve palsy is uncommon. ${ }^{1}$ This usually results from invasion by malignant thyroid tumours and is rarely observed with benign thyroid disease. ${ }^{1-3}$ No recorded case due to a distended oesophagus could be found.

Our patient had clear evidence of partial bilateral recurrent laryngeal nerve palsy in addition to tracheal compression due to distended oesophagus. The immobility of vocal cords in our patient was

\section{References}

1. Lawson, V.G. The management of airway involvement in thyroid tumours. Arch Otolaryngol 1983, 109: 86-90.

2. Holl-Allen, R.T.J. Laryngeal nerve paralysis and benign thyroid disease. Arch Otolaryngol 1967, 84: 335-337. not due to fixation of the arytenoids by the distended oesophagus as the cords were passively mobile at endoscopy.

It is difficult to explain the rapidity of the onset of bilateral nerve palsy and sudden relief of symptoms with the evacuation of the oesophagus. Rapid distension of the oesophagus with sudden build up of pressure within the confines of pretracheal fascia sufficient to impair neural microcirculation, seems the most likely explanation.

We would like to alert clinicians to this unusual but life-threatening complication of upper airways obstruction due to distended oesophagus by the features of preceding dysphagia and the X-ray appearance of oesophageal distension. In such cases oesophagogastroscopy and evacuation of the oesophageal contents is proposed as an emergency procedure.

3. Gani, J.S. \& Morrison, J.M. Simple thyroid cyst: cause of acute bilateral recurrent laryngeal nerve palsy. $\mathrm{Br}$ Med J 1987, 294: 1128-1129. 